Supporting Information

\title{
Structural dynamics of adsorption equilibrium for iodine adsorbed on $\mathrm{Au}(111)$
}

Masashi Nakamura ${ }^{*}{ }^{\dagger}$ Yuma Tanaka,${ }^{\dagger}$ Masamitu Takahasi, ${ }^{\ddagger}$ Hiroo Tajiri, ${ }^{\S}$ Osami Sakata, $₫$ Nagahiro Hoshi

$\dagger$ Department of Applied Chemistry and Biotechnology, Graduate School of Engineering, Chiba University, Yayoi-cho 1-33, Inage-ku, Chiba 263-8522, Japan.

* Institute for Advanced Synchrotron Light Source, National Institutes for Quantum and Radiological Science and Technology (QST), Kouto 1-1-1, Sayo-gun, Hyogo 679-5148, Japan.

$\S$ Research and Utilization Division, Japan Synchrotron Radiation Research Institute/SPring-8, Kouto 1-1-1, Sayo-gun, Hyogo 679-5148, Japan.

ף Synchrotron X-ray Group and Synchrotron X-ray station at SPring-8, National Institute for Materials Science (NIMS), Kouto 1-1-1, Sayo-gun, Hyogo 679-5148, Japan. 


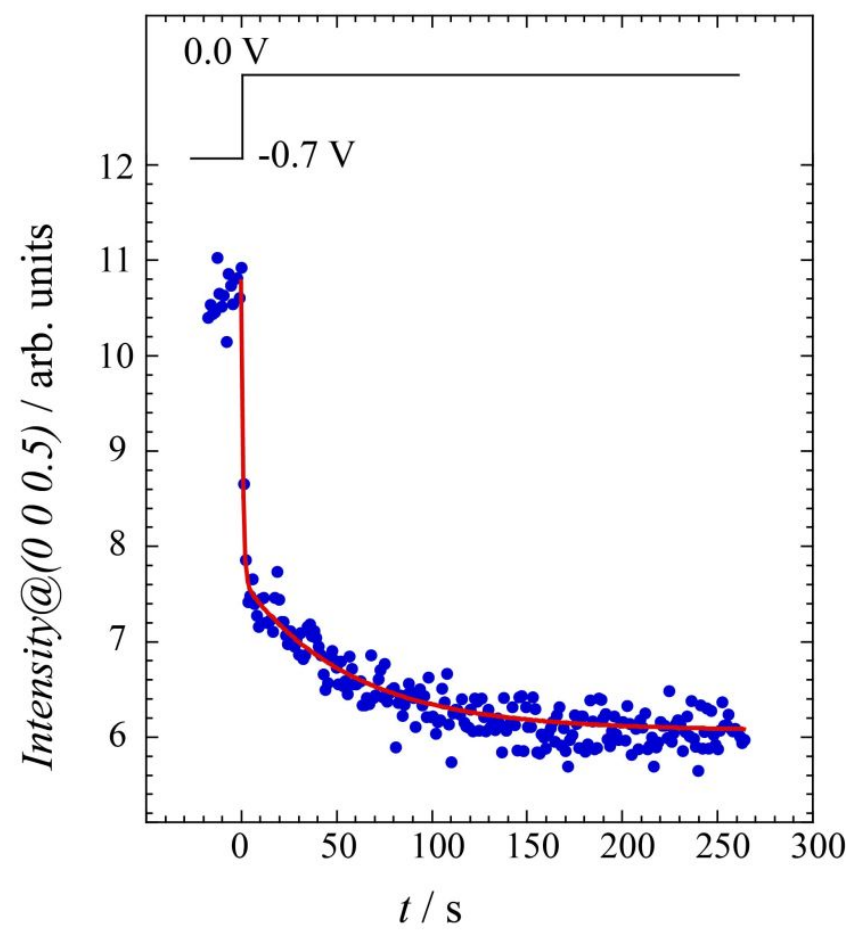

Figure S1. Transient X-ray diffraction at the peak position of the 000.5 . The solid line shows the best fit of the plots using a two-term exponential function. The time constant of slow decay is $57 \mathrm{~s}$. 